\title{
Systemic therapy for previously untreated advanced BRAF-mutated melanoma: navigating a shifting landscape
}

\author{
"Given the rapid changes in [BRAF-mutated melanoma], with \\ promising treatment options on the horizon, head-to-head trials \\ examining efficacy, safety and cost-effectiveness will be crucial for \\ treatment and funding decisions in the future."
}

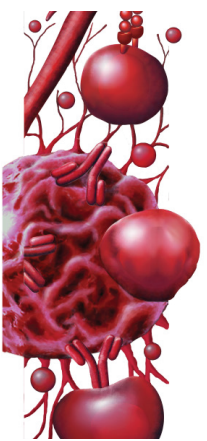

First draft submitted: 28 February 2017; Accepted for publication: 2 March 2017; Published online: 30 March 2017

\section{Keywords: BRAF mutation $\bullet$ immune checkpoint inhibitor $\bullet$ melanoma $\bullet$ targeted therapy}

Treatment of advanced cutaneous melanoma has rapidly evolved over recent years. This aggressive form of skin cancer, when not amenable to surgical resection due to locoregional or distant spread of tumor, has been associated with very poor prognosis [1]. Until recently, advanced melanoma was considered a fatal diagnosis with very few exceptions. Historically, high-dose IL-2 was offered to highly selected patients with limited comorbidity and excellent functional status for a small chance of durable disease control; however, this therapy is associated with severe, sometimes, life-threatening toxicity [2]. Cytotoxic chemotherapy was the standard of care for decades despite a lack of evidence indicating survival benefit [3].

Two major advances in this field have revolutionized the treatment landscape. With the development of molecularly targeted agents and immunotherapies, chemotherapy has largely been replaced as a first-line systemic treatment. Mutation in the BRAF (V600) gene has been identified as a highly prevalent oncogene leading to constitutive activation of the RAF/MEK/ERK cell signaling pathway [4]. Present in 40-60\% of cutaneous melanoma cases $[4,5]$, the protein product of this oncogene can be selectively targeted to arrest malignant cell growth. Targeted therapeutic agents including the tyrosine kinase inhibitors vemurafenib and dabrafenib have shown significant antineoplastic activity and clinical benefit for patients with advanced, $B R A F$-mutated melanoma [6,7]. Clinical responses with these small-molecule agents, however, are of limited duration, in part due to reactivation of the MAPK pathway leading to drug resistance [8]. Additionally, secondary skin malignancies can develop due to paradoxical activation of this pathway in cells with nonmutated BRAF [9]. Specific inhibitors of the MEK protein (which lies downstream of BRAF) including trametinib and cobimetinib have been developed, and the former has shown some clinical benefit as monotherapy [10]. The combination of BRAF and MEK inhibition, as compared with single-agent inhibition, has shown prolonged responses by delaying drug resistance and decreased incidence of skin-related adverse events $[8,11]$. Thus, the repertoire of targeted therapies has expanded.

The second breakthrough was the discovery of immune-checkpoints or co-regulatory molecules responsible for dampening immune response. Mechanisms to suppress T-cell activation are important to avoid autoimmunity after an infectious insult, yet these protective mechanisms can be exploited by tumor cells to avoid immune attack. In fact, immune evasion is now recognized as one of the hallmarks of cancer. Cytotoxic T-lymphocyte antigen-4 (CTLA-4) and programmed celldeath protein 1 (PD-1) have been characterized as important immune-checkpoints $[12,13]$.
Oren Levine ${ }^{1,2}$, Tahira Devji ${ }^{1}$ \& Feng $\mathrm{Xie}^{*}, 1,3$

'Department of Health Research Methods, Evidence, \& Impact, McMaster University, Room H306 Martha Wing, St Joseph's Healthcare Hamilton, 50 Charlton Ave. E. Hamilton, ON L8N 4A6, Canada

${ }^{2}$ Department of Oncology, McMaster University, Hamilton, ON, Canada ${ }^{3}$ Father Sean O'Sullivan Research Centre, St Joseph's Healthcare Hamilton, Hamilton, ON, Canada *Author for correspondence: Tel.: +1 9055221155 (ext: 35808) Fax: +1 9053087212 fengxie@mcmaster.ca 
Immunomodulatory monoclonal antibodies have been developed to interfere with the activity of CTLA- 4 (ipilimumab and tremelimumab) and PD-1 (nivolumab and pembrolizumab), allowing T-cell activation and proliferation with anticancer effect. These therapeutic agents have shown survival benefit for advanced melanoma regardless of $B R A F$ mutation status [14,15]. Monotherapy with an immune-checkpoint inhibitor has improved survival compared with chemotherapy [16]. The combination of CTLA- 4 and PD-1-directed therapy compared with monotherapy has shown promise in early outcomes, and overall survival data are eagerly awaited [17].

The development of targeted and immune-checkpoint inhibitors for advanced melanoma has occurred in parallel. Numerous novel treatments have shown clinical benefit in high-quality randomized controlled trials (RCTs), and many have entered routine clinical practice after receiving regulatory approval. For treatment-naive patients with advanced $B R A F$-mutated melanoma, there are now multiple effective treatment options available. The lack of head-to-head comparisons between targeted and immunotherapies, however, has left patients and clinicians without evidence to inform decisions on optimal first-line systemic treatment.

In some circumstances, clinical factors dictate treatment choice. For example, in the face of rapidly advancing visceral disease, high disease-related symptom burden or poor performance status due to tumor burden, targeted therapy may be favored for rapid disease stabilization [18]. Clinical experience has shown that such patients can experience swift recovery, but tumor response may be short-lived. In these patients, immunotherapy is not ideal because response is expected to have slower onset. Additionally, there is risk of pseudoprogression with tumors temporarily increasing in size before response occurs [19]. In the setting of brain metastases, small-molecule targeted therapies were thought to cross the blood-brain barrier more easily than monoclonal antibodies and, therefore, targeted therapies were previously favored over immunotherapies in this patient population. Mounting evidence suggests both targeted and immune-checkpoint inhibitors can be effective in this setting, contributing to the uncertainty in choosing first-line treatment for this group of patients [20,21].

In the absence of a compelling clinical circumstance to guide choice of treatment, patient-important factors must be considered. Targeted therapies are oral medications taken continuously until progression with the potential to impair quality of life due to daily side effects including skin-related adverse events, fatigue and fever. Immune-checkpoint inhibitors, administered as intermittent infusions, may not cause daily symptoms and side effects, but have the potential to cause severe autoimmune toxicities, most notably, colitis. With more widespread use of such agents, a wide variety of immune-related adverse events have been documented, affecting almost any organ system in the body. Such toxicities often have delayed onset and can be life-threatening if not identified and managed appropriately [22].

Despite the toxicity risk, there has been much excitement about the durable remissions seen with use of immunotherapies. Around one in five patients seems to achieve long-term disease control with use of ipilimumab [23], and it is hoped that combined CTLA-4/ PD-1-directed treatment will further improve patientimportant outcomes. This represents a major paradigm shift for prognostication of advanced melanoma. Moreover, the unprecedented survival outcomes for melanoma patients have contributed to the fervor with which immune-checkpoint inhibitors are being tested in other cancer types. Successes have already been achieved in non-small-cell lung cancer, urothelial bladder cancer and renal cell carcinoma, among other tumor types [24-26]. Many trials are currently underway which could lead to additional new indications for immune-checkpoint inhibitors. With respect to $B R A F$-mutated melanoma, however, recently published long-term data from early clinical trials suggest that a proportion of patients treated in first line with targeted therapy also achieved durable disease control, adding to the uncertainty of optimal therapy [27].

It is unlikely that additional RCTs will be carried out for head-to-head comparison of single agent targeted or immunotherapies currently available for treatment-naive patients. Rather, the focus of current inquiry is combinatorial strategies aiming to improve outcomes for melanoma patients by layering multiple drugs with potentially synergistic mechanisms of action. For instance, the combination of ipilimumab and bevacizumab, an antiangiogenic agent, is under investigation in a clinical trial (NCT01950390). A randomized Phase II trial has shown benefit of combined CTLA-4 inhibition and GM-CSF, a cytokine which augments antigen presentation and mitigates autoimmune toxicity [28]. A follow-up Phase II/III trial is testing the addition of GM-CSF to combined ipilimumab and nivolumab (NCT02339571). Optimal treatment sequence is another area of uncertainty, and this may be clarified by a current Phase III study that involves combined ipilimumab and nivolumab followed by combined dabrafenib and trametinib at progression, or the reverse treatment sequence (NCT02224781). Concurrent use of targeted therapies and a novel immunecheckpoint inhibitor is also the focus of a Phase III 
trial (NCT02908672). With numerous combinations under investigation, there is optimism that a greater proportion of patients will derive long-term benefit, yet the treatment landscape may become increasingly crowded as a result.

The recent network meta-analysis (NMA) by Devji et al. identified and synthesized the evidence from 15 RCTs involving 6662 treatment-naive patients with advanced melanoma to draw comparison between targeted therapy and immune-checkpoint inhibitors [29]. In light of the uncertainty toward optimal first-line treatment for patients with $B R A F$-mutated melanoma, this NMA estimated relative efficacy and safety for all possible pairwise comparisons between the included treatment options. Only treatment classes were compared since individual drug comparisons are too few to yield a robust analysis. This NMA found that PD-1 inhibition or combined BRAF/MEK inhibition significantly improves overall survival compared with other treatments, with no significant difference detected between them (hazard ratio: 1.02 ; $95 \%$ credible interval: 0.72 1.45). The rate of serious adverse events is lowest for PD-1 inhibition or chemotherapy. Thus, this analysis of current evidence suggests that PD-1 is an optimal choice for treatment-naive patients who do not require a rapid tumor regression. Objective response is substantially more likely with BRAF/MEK inhibition compared with other treatments, which supports current clinical practice to offer targeted therapy in the first line for patients with bulky or highly symptomatic disease. Long-term results of landmark trials of combined CTLA-4/PD-1 inhibition are awaited. While this approach may have

\section{References}

1 Statistics CCSSaCOC. Canadian Cancer Statistics 2016 (2016). www.cancer.ca/en/cancer-information/cancertype/skin-melanoma/statistics/?region $=$ on

2 Atkins MB, Kunkel L, Sznol M, Rosenberg SA. Highdose recombinant interleukin-2 therapy in patients with metastatic melanoma: long-term survival update. Cancer J. Sci. Am. 6(Suppl. 1) S11-S14 (2000).

3 Agarwala SS. Current systemic therapy for metastatic melanoma. Expert Rev. Anticancer Ther. 9(5), 587-595 (2009).

4 Davies H, Bignell GR, Cox C et al. Mutations of the BRAF gene in human cancer. Nature 417(6892), 949-954 (2002).

5 Curtin JA, Fridlyand J, Kageshita T et al. Distinct sets of genetic alterations in melanoma. N. Engl. J. Med. 353(20), 2135-2147 (2005).

6 Chapman PB, Hauschild A, Robert C et al. Improved survival with vemurafenib in melanoma with BRAF V600E mutation. N. Engl. J. Med. 364(26), 2507-2516 (2011).

7 Hauschild A, Grob JJ, Demidov LV et al. Dabrafenib in BRAF-mutated metastatic melanoma: a multicentre, open- the potential to further improve survival, the NMA found higher risk of serious adverse events with this combination [29].

Many of the novel therapies in treatment of advanced melanoma are costly. Particularly with combinations of multiple therapeutic agents, the financial burden is significant. In a resource-restricted healthcare environment, cost of care is an important factor in decisionmaking for patients and healthcare organizations. Cost-effectiveness of current treatment options is an area where more evidence is needed.

While the expanding armamentarium of systemic treatments for advanced, $B R A F$-mutated melanoma is encouraging for patients and clinicians, with each new drug approval, clinical decision-making becomes more complex. NMA of current evidence has provided a road map to navigate the evolving landscape of melanoma treatment. Given the rapid changes in this field, with promising treatment options on the horizon, head-to-head trials examining efficacy, safety and cost-effectiveness will be crucial for treatment and funding decisions in the future.

\section{Financial \& competing interests disclosure}

The authors have no relevant affiliations or financial involvement with any organization or entity with a financial interest in or financial conflict with the subject matter or materials discussed in the manuscript. This includes employment, consultancies, honoraria, stock ownership or options, expert testimony, grants or patents received or pending, or royalties.

No writing assistance was utilized in the production of this manuscript.

label, Phase 3 randomised controlled trial. Lancet 380 (9839), 358-365 (2012).

8 Robert C, Karaszewska B, Schachter J et al. Improved overall survival in melanoma with combined dabrafenib and trametinib. N. Engl. J. Med. 372(1), 30-39 (2015).

9 Hatzivassiliou G, Song K, Yen I et al. RAF inhibitors prime wild-type RAF to activate the MAPK pathway and enhance growth. Nature 464(7287), 431-435 (2010).

10 Flaherty KT, Robert C, Hersey P et al. Improved survival with MEK inhibition in BRAF-mutated melanoma. N. Engl. J. Med. 367(2), 107-114 (2012).

11 Larkin J, Ascierto PA, Dréno B et al. Combined vemurafenib and cobimetinib in BRAF-mutated melanoma. N. Engl. J. Med. 371(20), 1867-1876 (2014).

12 Topalian SL, Hodi FS, Brahmer JR et al. Safety, activity, and immune correlates of anti-PD-1 antibody in cancer. N. Engl. J. Med. 366(26), 2443-2454 (2012).

13 O'day SJ, Hamid O, Urba WJ. Targeting cytotoxic T-lymphocyte antigen-4 (CTLA-4): a novel strategy for the treatment of melanoma and other malignancies. Cancer 110 (12), 2614-2627 (2007). 
14 Shahabi V, Whitney G, Hamid O et al. Assessment of association between BRAF-V600E mutation status in melanomas and clinical response to ipilimumab. Cancer Immunol. Immunother. 61(5), 733-737 (2012).

15 Larkin J, Lao CD, Urba WJ et al. Efficacy and safety of nivolumab in patients with BRAF V600 mutant and BRAF wild-type advanced melanoma: a pooled analysis of 4 clinical trials. JAMA Oncol. 1(4), 433-440 (2015).

16 Robert C, Long GV, Brady B et al. Nivolumab in previously untreated melanoma without BRAF mutation. N. Engl. J. Med. 372(4), 320-330 (2015).

17 Larkin J, Chiarion-Sileni V, Gonzalez R et al. Combined nivolumab and ipilimumab or monotherapy in untreated melanoma. N. Engl. J. Med. 373(1), 23-34 (2015).

18 Dummer R, Hauschild A, Lindenblatt N, Pentheroudakis G, Keilholz U, Committee EG. Cutaneous melanoma: ESMO Clinical Practice Guidelines for diagnosis, treatment and follow-up. Annal. Oncol. 26(Suppl. 5) v126-v132 (2015).

19 Wolchok JD, Hoos A, O'day S et al. Guidelines for the evaluation of immune therapy activity in solid tumors: immune-related response criteria. Clin. Cancer Res. 15(23), 7412-7420 (2009).

20 Margolin K, Ernstoff MS, Hamid O et al. Ipilimumab in patients with melanoma and brain metastases: an open-label, Phase 2 trial. Lancet Oncol. 13(5), 459-465 (2012).

21 Long GV, Trefzer U, Davies MA et al. Dabrafenib in patients with Val600Glu or Val600Lys BRAF-mutant melanoma metastatic to the brain (BREAK-MB): a multicentre, openlabel, Phase 2 trial. Lancet Oncol. 13(11), 1087-1095 (2012).

22 Fecher LA, Agarwala SS, Hodi FS, Weber JS. Ipilimumab and its toxicities: a multidisciplinary approach. Oncologist
18(6), 733-743 (2013).

23 Schadendorf D, Hodi FS, Robert C et al. Pooled analysis of long-term survival data from Phase II and Phase III trials of ipilimumab in unresectable or metastatic melanoma. J. Clin. Oncol. 33(17), 1889-1894 (2015).

24 Brahmer J, Reckamp KL, Baas P et al. Nivolumab versus docetaxel in advanced squamous-cell non-small-cell lung cancer. N. Engl. J. Med. 373(2), 123-135 (2015).

25 Motzer RJ, Escudier B, Mcdermott DF et al. Nivolumab versus everolimus in advanced renal-cell carcinoma. N. Engl. J. Med. 373(19), 1803-1813 (2015).

26 Sharma P, Callahan MK, Bono P et al. Nivolumab monotherapy in recurrent metastatic urothelial carcinoma (CheckMate 032): a multicentre, open-label, two-stage, multi-arm, Phase 1/2 trial. Lancet Oncol. 17(11), 1590-1598 (2016).

27 Long GV, Weber JS, Infante JR et al. Overall survival and durable responses in patients with $B R A F$ V600-mutant metastatic melanoma receiving dabrafenib combined with trametinib. J. Clin. Oncol. 34(8), 871-878 (2016).

28 Hodi FS, Lee S, Mcdermott DF et al. Ipilimumab plus sargramostim vs ipilimumab alone for treatment of metastatic melanoma: a randomized clinical trial. JAMA 312(17), 1744-1753 (2014).

29 Devji T, Levine O, Neupane B, Beyene J, Xie F. Systemic therapy for previously untreated advanced $B R A F$-mutated melanoma: a systematic review and network meta-analysis of randomized clinical trials. JAMA Oncol. doi:10.1001/ jamaoncol.2016.4877 (2016) (Epub ahead of print). 